\title{
Upgrading on radical prostatectomy specimens of very low- and low-risk prostate cancer patients on active surveillance: A population-level analysis
}

Rashid K. Sayyid, MD, MSc${ }^{1}$; Brandon Wilson, BSN ${ }^{2}$; John Z. Benton, BS ${ }^{2}$; Atul Lodh, BS ${ }^{2}$; Eric F. Thomas, MD, MS ${ }^{1}$; Hanan Goldberg, MD, MSc ${ }^{3}$; Rabii Madi, MD, MBA ${ }^{1,4}$; Martha K. Terris, MD*1,4; Christopher J.D. Wallis, MD, PhD, FRCSC $^{* 5}$; Zachary Klaassen, MD, MSc ${ }^{1,4}$

${ }^{1}$ Section of Urology, Department of Surgery, Medical College of Georgia-Augusta University, Augusta, GA, United States; ${ }^{2}$ Medical College of Georgia, Augusta, GA, United States; ${ }^{3}$ Department of Urology, State University of New York Upstate, Syracuse, NY, United States; ${ }^{4} \mathrm{Georgia}$ Cancer Center, Augusta, GA, United States; ${ }^{5}$ Department of Urology, Vanderbilt University, Nashville, TN, United States

${ }^{*}$ Co-senior authors

Cite as: Sayyid RK, Wilson B, Benton JZ, et al. Upgrading on radical prostatectomy specimens of very low- and low-risk prostate cancer patients on active surveillance: A population-level analysis. Can Urol Assoc J 2020 December 15; Epub ahead of print. http://dx.doi.org/10.5489/cuaj.6868

Published online December 15, 2020

$* * *$

Abstract format accepted as a poster presentation at the Southeastern Sectional Meeting of the American Urological Association in New Orleans, LA, on March 21, 2020

\section{Abstract}

Introduction: A proportion of prostate cancer ( $\mathrm{PCa}$ ) patients initially managed with active surveillance (AS) are upgraded to a higher Gleason score (GS) at the time of radical prostatectomy $(\mathrm{RP})$. Our objective was to determine predictors of upgrading on RP specimens using a national database.

Methods: The Surveillance, Epidemiology, and End Results Prostate with Watchful Waiting database was used to identify AS patients diagnosed with very low- or lowrisk PCa who underwent delayed RP between 2010 and 2015. The primary outcome was upgrading to GS 7 disease or worse. Logistic regression analyses were used to evaluate demographic and oncological predictors of upgrading on final specimen. Results: A total of 3775 men underwent RP after a period of AS, 3541 (93.8\%) of whom were cT2a; 792 (21.0\%) patients were upgraded on RP specimen, with $85.4 \%$, $10.6 \%$, and $3.4 \%$ upgraded to GS $7(3+4), 7(4+3)$, and 8 diseases, respectively. On multivariable analysis, higher prostate-specific antigen (PSA) at diagnosis (5-10 vs. 
$0-2 \mathrm{ng} / \mathrm{ml}$, odd ratio [OR] 2.59, $\mathrm{p}<0.001)$ and percent core involvement $(80-100 \%$ vs. $0-20 \%$, OR $2.52, \mathrm{p}=0.003$ ) were significant predictors of upgrading on final RP specimen, whereas higher socioeconomic status predicted lower odds of upgrading (highest vs. lowest quartile OR $0.75, \mathrm{p}=0.013$ ).

Conclusions: Higher baseline PSA and percent positive cores involvement are associated with significantly increased risk of upgrading on RP after AS, whereas higher socioeconomic status predicts lower odds of such events. These results may help identify patients at increased risk of adverse pathology on final specimen who may benefit from earlier definitive treatment.

\section{Introduction}

Active surveillance (AS) has emerged as the preferred management option for patients with favorable risk, localized prostate cancer (PCa) (1). While AS reduces the risk of overtreatment of clinically insignificant $\mathrm{PCa}$, at least a third of such patients will undergo definitive treatment within 10 years of diagnosis (2). Amongst patients undergoing radical prostatectomy (RP), a significant number are diagnosed with more histologically aggressive forms of $\mathrm{PCa}$ (3-5), which is likely due to a combination of disease progression and/or fallibility of initial risk stratification approaches. As higher-grade disease portends worse oncologic outcomes (6), identifying patients at increased risk of worse pathologic disease becomes critical as such patients may be considered for earlier definitive treatment. Our objective was thus to identify predictors of upgrading on radical prostatectomy specimens in men with very low and low-risk prostate cancer initially opting for AS using a nationally representative cohort.

\section{Methods}

\section{Study design, setting, and participants}

We utilized the Surveillance, Epidemiology, and End Results (SEER) Prostate with Watchful Waiting database, which is a nationally representative database supported by the National Cancer Institute. This database captures men with incident PCa from 18 population-based registries, between 2010 and 2015, and covers approximately $30 \%$ of the US population (7). We included men younger than 80 years old with National Comprehensive Cancer Network very low- or low-risk PCa (8), who opted initially for AS and ultimately underwent an RP. No institutional review board approval was required for this study.

\section{Study outcome}

The primary study outcome was upgrading to Gleason Score (GS) 7 (3+4) or worse on final pathologic specimen. Other pathologic information such as stage, 
extraprostatic extension, or percent tumor involvement was not available from this database.

\section{Study variables}

The following patient-level variables were abstracted: year of diagnosis, age at diagnosis, race, insurance status, marital status, SEER registry region, first cancer diagnosis (i.e. whether PCa was the first cancer diagnosis the patient received or whether he had previously been diagnosed with a different, unrelated malignancy), cT stage (cT1a-b, cT1c, or cT2a), prostate-specific antigen (PSA) level at diagnosis (0-2, $2-5$, or $5-10 \mathrm{ng} / \mathrm{ml}$ ), GS on prostate biopsy or transurethral resection of prostate, and number of positive and examined prostate cores/specimens. County-level socioeconomic status $\left[1^{\text {st }}\right.$ (lowest) $, 2^{\text {nd }}, 3^{\text {rd }}$, or $4^{\text {th }}$ (highest) $]$ was derived from: percentage of individuals (i) with less than a high school education, (ii) below the poverty line, (iii) unemployed, (iv) foreign born, and (v) median household income) (9). Percent positive cores variable was derived from number of cores/specimen positive and examined.

\section{Statistical methods}

Continuous variables were reported using medians and interquartile ranges (IQR), with categorical variables described using frequencies and proportions. Univariable and multivariable logistic regression analyses were performed to evaluate for predictors of upgrading on RP specimen. All predictors were operationalized as categorical variables in the regression models. The overall statistical significance of categorical variables was assessed using the likelihood ratio test. Due to their clinical relevance, decision was made a priori to include all the study variables in both the univariable and multivariable models. Variable collinearity was evaluated using the variance inflation factor test. Statistical significance was set at $p<0.05$. All statistical analyses were performed using R version 3.6.1 (The R Foundation for Statistical Computing, Vienna, Austria).

\section{Results}

Figure 1 demonstrates the derivation of the study cohort. Amongst 32,874 men with very low- or low-risk PCa, 3,775 (11.5\%) underwent RP during the study period. Median patient age was 60.0 years (IQR 55.0-65.0). 2,774 (73.5\%) patients were Caucasian, 2,981 (79.0\%) married, 3,548 (94.0\%) were insured, and $110(2.9 \%)$ covered by Medicaid (Table 1). 3,765 (99.7\%) were GS 6 on biopsy/TURP specimen, with the remaining $10(0.3 \%)$ GS 5 or lower, and 3,541 (93.8\%) were clinical stage cT2a. Median PSA and percent positive cores were $4.9 \mathrm{ng} / \mathrm{ml}$ (IQR 3.7-6.3) and $15.4 \%$ (IQR 8.3\%-25.0\%), respectively. PCa was the first cancer diagnosis in 3,590 $(95.1 \%)$ of patients.

$792(21.0 \%)$ patients were upgraded on final RP specimen. $676(85.4 \%)$ were upgraded to GS $7(3+4), 84(10.6 \%)$ to GS $7(4+3)$, and $27(3.4 \%)$ to GS 8 . There were 
no cases of upgrading to GS 9 or worse (Table 2). 761 (96.1\%) patients had cT2a disease compared to 31 (3.9\%) with non-palpable disease at diagnosis. Of 28,613 patients with non-palpable disease, only $55(0.2 \%)$ underwent RP and $20(36.4 \%)$ of those patients were upgraded on final pathology specimen.

On univariable analysis, year of diagnosis (2014-2015 vs. 2010-2011 odds ratio [OR] 1.44, $\mathrm{p}<0.001$ ), age at diagnosis (70-79 vs. 30-49 OR 1.97, $\mathrm{p} 0.002)$, T stage (overall variable significance $\mathrm{p}$ 0.018), PSA at diagnosis (5-10 vs. 0-2 ng/ml OR $2.07, \mathrm{p}<0.001)$, percent positive cores ( $80-100 \%$ vs $0-20 \%$ OR $2.50, \mathrm{p} 0.002$ ), and SES (highest vs. lowest OR $0.75, \mathrm{p} 0.007$ ) were significantly associated with risk of upgrading on final RP specimen.

On multivariable analysis, higher PSA at diagnosis (5-10 vs. 0-2 ng/ml OR $2.38, \mathrm{p} 0.003$ ) and percent core involvement ( $80-100 \%$ vs. $0-20 \%$ OR $3.20, \mathrm{p}<0.001)$ were significant predictors of upgrading on final RP specimen. Higher SES was associated with a lower risk of upgrading (highest vs. lowest OR 0.66, p 0.013).

\section{Discussion}

In this nationally representative cohort of men with very low- and low-risk PCa initially managed with AS, we demonstrate that among men eventually opting for RP, $21.0 \%$ are upgraded to GS $7(3+4)$ disease or worse. On multivariable logistic regression analysis, we demonstrate that increasing PSA and higher percent positive cores are significant predictors of increased odds of upgrading on final pathologic specimen, which is consistent with results published in the literature. ${ }^{2-5}$ Conversely, patients of higher SES are at lower risk of upgrading on final pathologic specimen. Notably, older age and African American race were not significant predictors of upgrading in our cohort after adjusting for demographic and oncologic variables on multivariable analysis. This is in contrast to previous studies demonstrating their prognostic utility. ${ }^{10,11}$

This observed difference is likely due to two important reasons. The underlying nature of this cohort is distinct from that of other reported series. Whereas this is a cohort of patients who were initially managed with AS and subsequently opted for treatment after at least a year of surveillance, most previous studies report on the outcomes of PCa patients with AS-eligible disease, but who did not necessarily undergo AS..$^{3,4,5,11}$ This important difference leads to underlying selection biases that create two unique cohorts. Thus results from previous studies are not necessarily translatable to this cohort. Second, it is important to emphasize that multivariable analysis allows us to control for the effect of confounders. The SEER Prostate Watchful Waiting database contains a broad range of patient-level demographic and oncologic variables, which is critical to minimize the impact of unknown confounders. Older age was predictive of increased risk of upgrading on our univariable analysis, however on multivariable analysis this was no longer significant after controlling for the effect of other demographic/oncologic variables. 
Notably, $93.8 \%$ of patients in our cohort of men undergoing RP were cT2a, which is significantly higher than that of our original cohort of 32,874 men with very low/low-risk PCa on AS (15.8\%). This finding is consistent with results from large AS cohorts which also demonstrated that $\geq \mathrm{cT} 2 \mathrm{a}$ was a predictor for intervention (OR $1.96, \mathrm{p}<0.001) .^{2}$ Although the exact reason for intervention was not available for our cohort, these findings suggest that palpable disease on rectal exam may trigger physician-level anxiety, subsequently prompting recommendation for intervention. This is consistent with previous literature suggesting that PCa patients' treatment decisions are largely based on urologists' recommendations and less on patients' personal views of the relative pros and cons of management alternatives.

Our data also demonstrates that lower SES is associated with higher risk of upgrading, even after controlling for known confounders such as age and race. These findings are likely secondary to underlying biases, which have not been accounted for in our analysis. Patients of lower SES are less likely to follow up with their physicians, ${ }^{13}$ and thus less likely to undergo serial PSA measurements, rectal exams and confirmatory biopsies as dictated by most AS regimens. ${ }^{2}$ This puts them at higher risk of being "under-staged" leading to a higher risk of adverse pathology on RP specimens.

Our study has several limitations. SEER Prostate with Watchful Waiting database only provides demographic and pathologic data at diagnosis. In other words, if a patient undergoes a confirmatory prostate biopsy with GS upgrading or has a significant rise in PSA on follow-up, this is not captured by the database.

Consequently, we are unable to discern the triggers for intervention for these patients who were very low- or low-risk at initial diagnosis. Furthermore, results from preoperative imaging tools (i.e. transrectal ultrasound, multiparametric magnetic resonance imaging [MRI]) and pathologic information (e.g. extent of core involvement, perineural invasion) were not available, ${ }^{14}$ and thus not accounted for in our regression analysis. The significance of this is further magnified by the fact that $93.8 \%$ of our cohort was cT2a at diagnosis. It would be expected that such patients would undergo subsequent imaging with multiparametric MRI prior to being placed on AS. This may potentially result in targeted repeat biopsies with results of that affecting subsequent decision to undergo definitive treatment with RP. This study is also limited by its retrospective nature and biases inherent to the use of health administrative databases.

\section{Conclusions}

93.8\% of patients with very low- and low-risk PCa initially managed with AS and opting for delayed RP are cT2a at diagnosis. $21.0 \%$ of such are subsequently upgraded to GS 7(3+4) disease or worse on RP specimen. Increasing PSA and higher percent positive cores involved are associated with a significantly increased risk of pathologic upgrading, whereas higher SES conversely predicts a lower risk of such an 
event. Notably, age at diagnosis and race are not associated with risk of upgrading after controlling for relevant demographic and oncologic patient variables. These results may help identify patients at increased risk of adverse pathology on final specimen who may benefit from earlier definitive treatment. 


\section{References}

1. Sanda MG, Cadeddu JA, Kirkby E, et al. Clinically Localized Prostate Cancer: AUA/ASTRO/SUO Guideline. Part I: Risk Stratification, Shared Decision Making, and Care Options. J Urol 2018;199: 683-690.

2. Klotz L, Vesprini D, Sethukavalan P, et al. Long-term follow-up of a large active surveillance cohort of patients with prostate cancer. $J$ Clin Oncol 2015;33(3):272-7.

3. Verep S, Erdem S, Ozluk Y, Lilicaslan I, Sanli O, Ozcan F. The pathological upgrading after radical prostatectomy in low-risk prostate cancer patients who are eligible for active surveillance: How safe is it to depend on bioptic pathology? Prostate 2019;79(13):1523-9.

4. Kaye DR, Qi J, Morgan TM, et al. Pathological upgrading at radical prostatectomy for patients with Grade Group 1 prostate cancer: implications of confirmatory testing for patients considering active surveillance. BJU Int 2019;123(5):846-53.

5. Vellekoop A, Loeb S, Folkvaljon Y, Stattin P. Population based study of predictors of adverse pathology among candidates for active surveillance with Gleason 6 prostate cancer. J Urol 2014;191(2):350-7.

6. Albertsen PC, Hanley JA, Fine J. 20-year outcomes following conservative management of clinically localized prostate cancer. JAMA 2005;293(17):2095-101.

7. National Cancer Institute. https://seer.cancer.gov/seerstat/databases/prostateww/index.html. Accessed February 18, 2020.

8. Carroll PH, Mohler JL. NCCN Guidelines Updates: Prostate Cancer and Prostate Cancer Early Detection. J Natl Compr Canc Netw 2018;16(5S):620-3.

9. Chandrasekar T, Klaassen Z, Goldberg H, Sayyid RK, Kulkarni GS, Fleshner NE. High competing risks minimize real-world utility of adjuvant targeted therapy in renal cell carcinoma: a population-based analysis. Oncotarget 2018;9(24): 16731-43.

10. Leeman JE, Chen M, Huland H, Graefen M, D'Amico AV, Tilki D. Advancing Age and the Odds of Upgrading and Upstaging at Radical Prostatectomy in Men With Gleason Score 6 Prostate Cancer. Clin Genitourin Cancer 2019;17(6):e1116-21.

11. Maurice MJ, Sundi D, Schaeffer EM, Abouassaly R. Risk of Pathological Upgrading and Up Staging Among Men With Low Risk Prostate Cancer Varies by Race: Results From the National Cancer Database. J Urol 2017;197(3 Pt 1),627-31.

12. Scherr KA, Fagerlin A, Scherer LD, et al. Physician Recommendations Trump Patient Preferences in Prostate Cancer Treatment Decisions. Med Decis Making 2017;137(1):56-69.

13. Wong MKY, Wang JT, Czarnecki A, et al. Factors associated with physician follow-up among patients with chest pain discharged from the emergency department. CMAJ 2015;187(5):E160-8. 
14. Briganti A, Fossati N, Catto JW, et al. Active Surveillance for Low-risk Prostate Cancer: The European Association of Urology Position in 2018. Eur Urol 2018;74(3):357-68. 


\section{Figures and Tables}

Fig. 1. Study flow chart. GS: Gleason score; PSA: prostate-specific antigen; SEER: Surveillance, Epidemiology, and End Results; WW: watchful waiting.

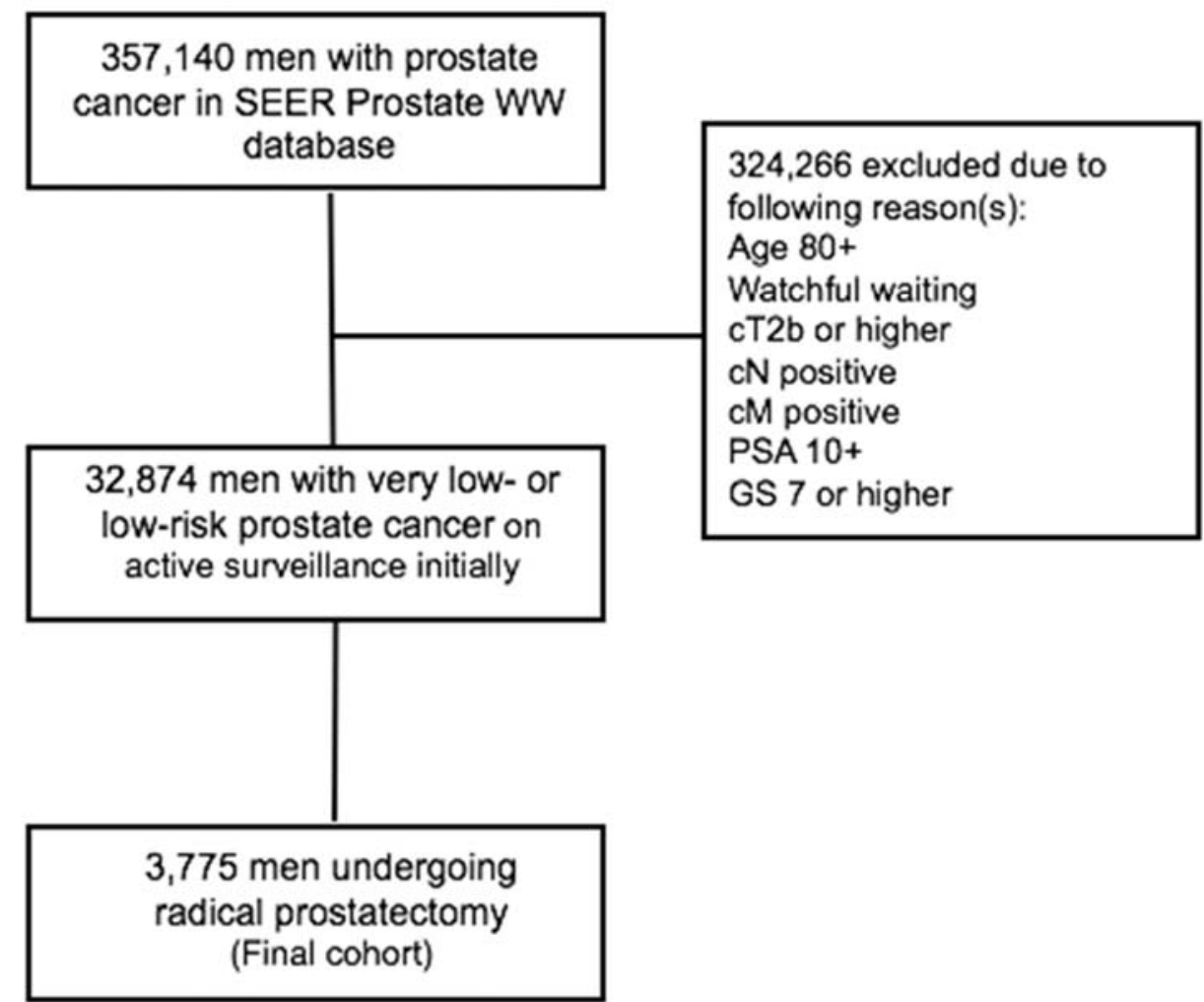




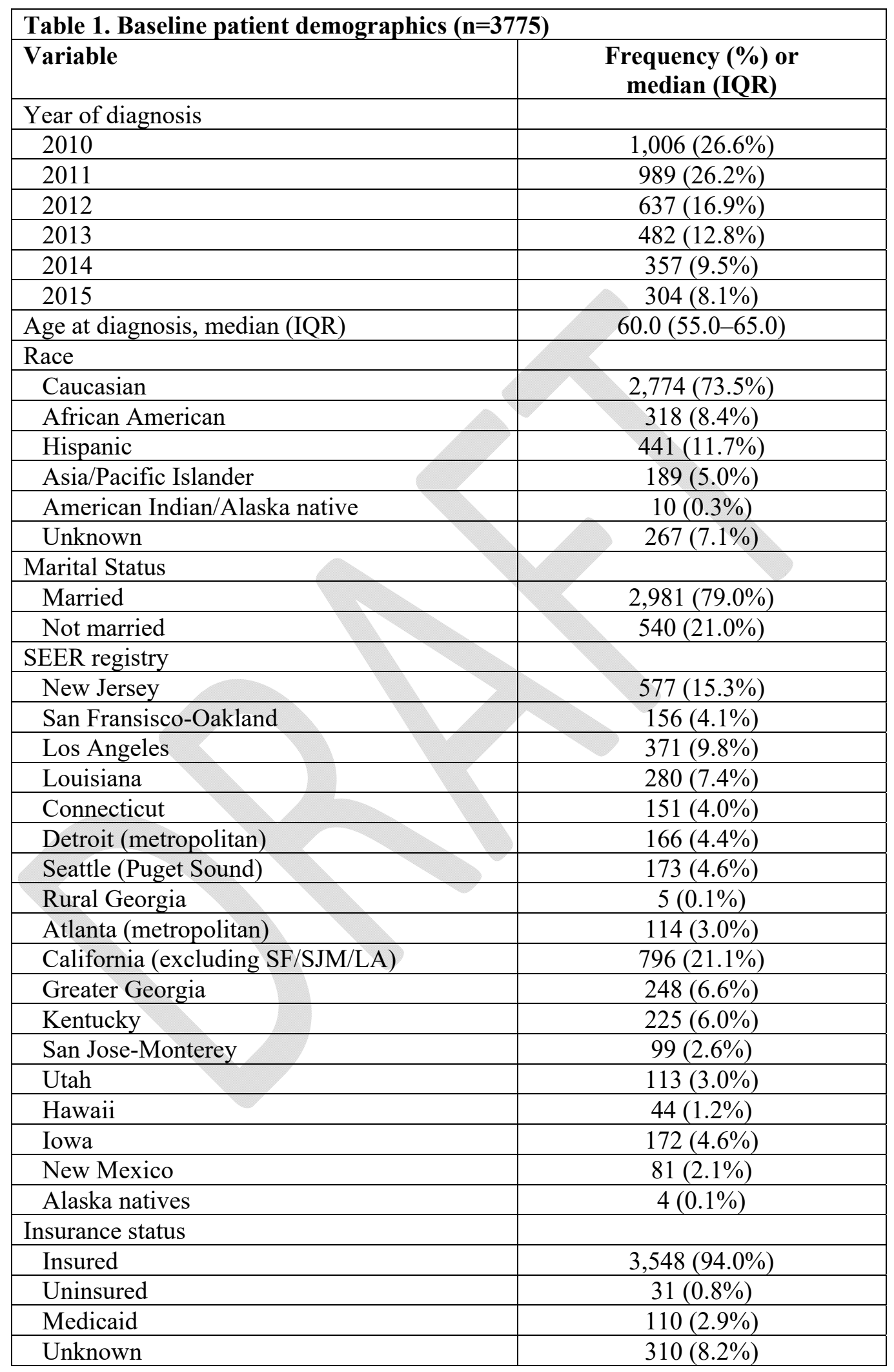




\begin{tabular}{|l|c|}
\hline Socioeconomic status & \\
\hline 1 (lowest) & $993(26.3 \%)$ \\
\hline 2 & $719(19.0 \%)$ \\
\hline 3 & $997(26.4 \%)$ \\
\hline 4 (highest) & $1066(28.2 \%)$ \\
\hline
\end{tabular}

IQR: interquartile range; SEER: Surveillance, Epidemiology, and End Results.

\begin{tabular}{|l|c|}
\hline \multicolumn{2}{|l|}{ Table 2. Study cohort oncological characteristics (n=3775) } \\
\hline Gleason score on biopsy/TURP & $10(0.3 \%)$ \\
\hline$<6$ & $3765(99.7 \%)$ \\
\hline 6 & \\
\hline Gleason Score on final pathological specimen & $44(1.2 \%)$ \\
\hline$<6$ & $2714(71.9 \%)$ \\
\hline 6 & $676(17.9 \%)$ \\
\hline $7(3+4)$ & $84(2.2 \%)$ \\
\hline $7(4+3)$ & $27(0.7 \%)$ \\
\hline 8 & $0(0 \%)$ \\
\hline $9-10$ & $230(6.1 \%)$ \\
\hline NA & $4.9(3.7-6.3)$ \\
\hline PSA at diagnosis, median (IQR) & $15.4 \%(8.3-25.0 \%)$ \\
\hline Percent cores positive, median (IQR) & $5(0.1 \%)$ \\
\hline cT Stage & $1(0.0 \%)$ \\
\hline cT1a & $228(6.0 \%)$ \\
\hline cT1b & $3541(93.8 \%)$ \\
\hline cT1c & $3590(95.1 \%)$ \\
\hline cT2a & $3185(4.9 \%)$ \\
\hline Prostate cancer as first diagnosed malignancy & \\
\hline Yes & \\
\hline No (i.e., previous, separate cancer diagnosis) & \\
\hline
\end{tabular}

IQR: interquartile range; PSA: prostate-specific antigen; TURP: transurethral resection of prostate. 


\begin{tabular}{|c|c|c|c|c|c|c|}
\hline Variable & OR & $95 \%$ CI & $\mathbf{p}$ & OR & $95 \% \mathrm{CI}$ & $\mathbf{p}$ \\
\hline $\begin{array}{l}\text { Year of diagnosis } \\
\text { (reference: } 2010- \\
11 \text { ) }\end{array}$ & & & $<0.001^{*}$ & & & 0.12 \\
\hline $2012-13$ & 1.06 & $0.88-1.27$ & 0.55 & 1.08 & $0.84-1.39$ & 0.55 \\
\hline $2014-15$ & 1.44 & $1.17-1.77$ & $<0.001^{*}$ & 1.24 & $0.92-1.67$ & 0.15 \\
\hline $\begin{array}{l}\text { Age at diagnosis } \\
\text { (reference: } 30-49 \\
\text { years) }\end{array}$ & & & $<0.001^{*}$ & & & 0.061 \\
\hline $50-59$ & 1.27 & $0.92-1.79$ & 0.16 & 1.28 & $0.80-2.11$ & 0.32 \\
\hline $60-69$ & 1.47 & $1.06-2.06$ & $0.023^{*}$ & 1.62 & $0.99-2.67$ & 0.051 \\
\hline $70-79$ & 1.97 & $1.30-3.02$ & $0.002^{*}$ & 1.44 & $0.76-2.76$ & 0.26 \\
\hline $\begin{array}{l}\text { Race (reference: } \\
\text { Caucasian) }\end{array}$ & & & 0.38 & & & 0.18 \\
\hline African American & 1.27 & $0.96-1.66$ & 0.094 & 1.34 & $0.90-1.95$ & 0.14 \\
\hline Hispanic & 0.98 & $0.76-1.25$ & 0.87 & 0.97 & $0.66-1.40$ & 0.85 \\
\hline $\begin{array}{l}\text { Asia/Pacific } \\
\text { Islander/America } \\
\text { n Indian/Alaska } \\
\text { Native }\end{array}$ & 1.11 & $0.77-1.56$ & 0.57 & 1.20 & $0.69-2.01$ & 0.49 \\
\hline $\begin{array}{l}\text { Insurance status } \\
\text { (reference: insured) }\end{array}$ & & & 0.90 & P & & 0.51 \\
\hline Uninsured & 1.22 & $0.48-2.77$ & 0.65 & 1.92 & $0.28-8.69$ & 0.43 \\
\hline Medicaid & 0.98 & $0.59-1.56$ & 0.95 & 1.15 & $0.60-2.10$ & 0.66 \\
\hline $\begin{array}{l}\text { Marital status } \\
\text { (reference: married) }\end{array}$ & & & 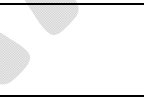 & & & \\
\hline Not married & 1.06 & $0.84-1.32$ & 0.62 & 0.98 & $0.72-1.33$ & 0.91 \\
\hline $\begin{array}{l}\text { PCa as first cancer } \\
\text { diagnosis } \\
\text { (reference: } \\
\text { previously } \\
\text { diagnosed with } \\
\text { other cancer) }\end{array}$ & 1.14 & $0.78-1.69$ & 0.52 & 1.05 & $0.62-1.86$ & 0.86 \\
\hline $\begin{array}{l}\text { SEER registry } \\
\text { region (reference: } \\
\text { Northeast) }\end{array}$ & & & 0.80 & & & 0.16 \\
\hline Southeast & 0.92 & $0.72-1.17$ & 0.49 & 0.73 & $0.49-1.07$ & 0.10 \\
\hline Midwest & 0.99 & $0.72-1.35$ & 0.93 & 1.18 & $0.75-1.83$ & 0.47 \\
\hline West & 1.01 & $0.82-1.26$ & 0.90 & 0.83 & $0.59-1.18$ & 0.29 \\
\hline $\begin{array}{l}\text { Socioeconomic } \\
\text { status }\end{array}$ & & & $0.049^{*}$ & & & 0.010 \\
\hline
\end{tabular}




\begin{tabular}{|c|c|c|c|c|c|c|}
\hline $\begin{array}{l}\text { (reference: } 1 \\
\text { ([lowest]) }\end{array}$ & & & & & & \\
\hline 2 & 0.81 & $0.64-1.02$ & 0.073 & 0.80 & $0.56-1.13$ & 0.20 \\
\hline 3 & 0.85 & $0.68-1.05$ & 0.12 & 0.91 & $0.6-1.23$ & 0.52 \\
\hline 4 (highest) & 0.75 & $0.60-0.92$ & $0.007^{*}$ & 0.66 & $0.47-0.91$ & $0.013^{*}$ \\
\hline $\begin{array}{l}\text { cT stage (reference: } \\
\text { T1a,b) }\end{array}$ & & & $0.018^{*}$ & & & 0.93 \\
\hline $\mathrm{cT} 1 \mathrm{c}$ & 1.71 & $\begin{array}{l}0.21- \\
35.56 \\
\end{array}$ & 0.65 & 18.6 & $0.0-76.2$ & 0.97 \\
\hline $\mathrm{cT} 2 \mathrm{a}$ & 0.84 & $\begin{array}{l}0.11- \\
17.07\end{array}$ & 0.88 & 75.0 & $0.21-102.7$ & 0.97 \\
\hline $\begin{array}{l}\text { PSA at diagnosis } \\
\text { (reference: } 0-2 \\
\mathrm{ng} / \mathrm{ml} \text { ) }\end{array}$ & & & $<0.001^{*}$ & & & $<0.001^{*}$ \\
\hline $2-5 \mathrm{ng} / \mathrm{ml}$ & 2.00 & $1.40-2.92$ & $<0.001^{*}$ & 2.63 & $1.54-4.83$ & $<0.001^{*}$ \\
\hline $5-10 \mathrm{ng} / \mathrm{ml}$ & 2.07 & $1.46-3.02$ & $<0.001^{*}$ & 2.38 & $1.39-4.36$ & $0.003^{*}$ \\
\hline $\begin{array}{l}\text { Percentage of } \\
\text { positive cores } \\
\text { (reference: } 0-20 \% \text { ) }\end{array}$ & & & $<0.001^{*}$ & & & $<0.001^{*}$ \\
\hline $20-40 \%$ & 2.21 & $1.72-2.85$ & $<0.001^{*}$ & 2.22 & $1.69-2.90$ & $<0.001^{*}$ \\
\hline $40-60 \%$ & 2.86 & $1.97-4.12$ & $<0.001^{*}$ & 2.74 & $1.83-4.08$ & $<0.001^{*}$ \\
\hline $60-80 \%$ & 1.88 & $0.81-3.99$ & 0.12 & 1.57 & $0.63-3.54$ & 0.30 \\
\hline $80-100 \%$ & 2.50 & $1.36-4.46$ & $0.002^{*}$ & 3.20 & $1.68-5.97$ & $<0.001^{*}$ \\
\hline
\end{tabular}

"Statistically significant. CI: Confidence interval; OR: odds ratio; PCa: prostate cancer; PSA: prostate-specific antigen; SEER: Surveillance, Epidemiology, and End Results. 